3. V. L. Klee, Extremal structure of convex sets. II, Math. Z. vol. 69 (1958) pp. 90104.

4. S. Mazur, Über konvexe Mengen in linearen normierten Räumen, Studia Math. vol. 4 (1933) pp. 70-84.

5. - Über schwach Konvergenz in den Räumen $\left(L^{p}\right)$, Studia Math. vol. 4 (1933) pp. 128-133.

6. R. R. Phelps, Subreflexive normed linear spaces, Arch. Math. vol. 8 (1957) pp. $444-450$. 169.

7. - Some subreflexive Banach spaces, Arch. Math. vol. 10 (1959) pp. 162-

INSTITUTE FOR ADVANCED STUDY

\title{
ISOMETRIES OF GROUP ALGEBRAS ${ }^{1}$
}

\section{PAUL CIVIN}

Let $G$ be a locally compact abelian group, $\hat{G}$ its character group, and $A$ the group algebra of $G$. Associated with any automorphism $\phi$ of $A[2]$ is a homeomorphism $\tau$ of $\hat{G}$ onto itself, with the property that, for $\alpha \in \hat{G}, f \in A$ and $\mathfrak{F}$ the Fourier transform, $\mathfrak{F}(\phi(f))(\tau \alpha)$ $=\mathfrak{F}(f)(\alpha)$. Results of Helson [2] and Wendel [4] state that if $e$ is the unit of $\hat{G}$, then

$$
\tau(e) \tau(x y)=\tau(x) \tau(y), \quad \text { for all } x, y \in \hat{G},
$$

if and only if $\phi$ is an isometry. The object of the present note is to give a further equivalent form of the statement that $\phi$ is an isometry.

Let $T_{\alpha}, \alpha \in \hat{G}$, be that operator on $A$ which for all $x \in G, f \in A$ satisfies $\left(T_{\alpha} f\right)(x)=f(x)(x, \alpha)$. We consider homomorphisms $\phi$ of $A$ onto $A$ such that to each $\alpha$ there is a $\rho(\alpha) \in \hat{G}$ such that

$$
\phi T_{\alpha}=T_{\rho(\alpha)} \phi, \quad \alpha \in \hat{G} .
$$

Our result is that such homomorphisms are isomorphisms and indeed isometries.

THEOREM 1. Let $\phi$ be a homomorphism of the group algebra $A$ of the locally compact abelian group $G$ onto itself. Suppose that $\phi$ satisfies (2); then $\phi$ is an isomorphism.

Let $K$ be the kernel of $\phi$. Since $\phi$ is automatically continuous [3], $K$ is a proper closed ideal of $A$. The Wiener Tauberian theorem thus yields a maximal regular ideal $M$ containing $K$. From condition (2) it follows that for $k \in K$ and $\beta \in \hat{G}, \phi T_{\beta}(k)=T_{\rho(\beta)} \phi(k)=0$, and there-

Received by the editors September 11, 1959.

1 This research was sponsored in part by the National Science Foundation under grant NSF-G5865. 
fore $T_{\beta}(K) \subset K \subset M$. Let $k \in K$ and let $\alpha$ be the character corresponding to $M$; then $0=\mathfrak{F}\left(T_{\beta}(k)\right)(\alpha)=\mathfrak{F}(k)(\beta \alpha)$ for arbitrary $\beta \in \hat{G}$. Consequently $k=0$, and $\phi$ is an isomorphism.

Lemma. Let $\phi$ be an automorphism of $A$ which satisfies (2), and let $\tau$ be the associated homeomorphism of $\hat{G}$ onto itself. Then:

(i) The mapping $\rho$ is a homomorphism of $\hat{G}$ onto itself.

(ii) For any $\alpha, \beta \in \hat{G}, \tau(\alpha \beta)=\rho(\beta) \tau(\alpha)$.

We show that $\rho$ is a homomorphism into $\hat{G}$. That the image of $\hat{G}$ under $\rho$ is all of $\hat{G}$ is then a consequence of (ii). For all $f \in A, T_{\rho(\alpha \beta)} \phi(f)$ $=\phi T_{\alpha \beta}(f)=\phi T_{\alpha} T_{\beta}(f)=T_{\rho(\alpha)} \phi T_{\beta}(f)=T_{\rho(\alpha)} T_{\rho(\beta)} \phi(f)$. Since $\phi(f)$ ranges over $A, T_{\rho(\alpha \beta)}=T_{\rho(\alpha)} T_{\rho(\beta)}$, whence $\rho(\alpha \beta)=\rho(\alpha) \rho(\beta)$.

It follows from (2) that for all $\beta \in \hat{G}, \mathfrak{F}\left(\phi T_{\alpha}(f)\right)(\tau(\beta))$ $=\mathfrak{F}\left(T_{\rho(\alpha)} \phi(f)\right)(\tau(\beta))$. Thus, $\mathfrak{F}(\phi(f))(\rho(\alpha) \tau(\beta))=\mathfrak{F}\left(T_{\rho(\alpha)} \phi(f)(\tau(\beta))\right.$ $=\mathfrak{F}\left(\phi T_{\alpha}(f)\right)(\tau(\beta))=\mathfrak{F}\left(T_{\alpha}(f)\right)(\beta)=\mathfrak{F}(f)(\alpha \beta)=\mathfrak{F}(\phi(f))(\tau(\alpha \beta))$. Since the Fourier transforms of elements in $A$ separate the points of $\hat{G}$, we deduce that $\rho(\alpha) \tau(\beta)=\tau(\alpha \beta)$.

THEOREM 2. Let $A$ be the group algebra of a locally compact abelian group $G$, and let $\phi$ be an automorphism of $A$. The following conditions are equivalent:

(a) $\phi$ is an isometry.

(b) The associated homeomorphism $\tau$ of $\hat{G}$ onto itself satisfies (1).

(c) $\phi$ satisfies (2).

In view of our earlier remarks it suffices to show that (b) is equivalent to (c). Suppose that $\phi$ satisfies (2). Let $\tau$ be the homeomorphism of $\hat{G}$ associated with $\phi$. From (ii) of the lemma, with the identity $e$ of $\hat{G}$ in the role of $\alpha$, we obtain $\tau(\beta)=\rho(\beta) \tau(e)$ for all $\beta \in \hat{G}$. Thus, using the above and (i) of the lemma,

$$
\tau(e) \tau(\alpha \beta)=(\tau(e))^{2} \rho(\alpha \beta)=\tau(e) \rho(\alpha) \tau(e) \rho(\beta)=\tau(\alpha) \tau(\beta) .
$$

Hence (c) implies (b).

Suppose that $\tau$ satisfies (1). Recall that

$$
\mathfrak{F}(\phi(f))(\tau(\alpha))=\mathfrak{F}(f)(\alpha), \quad \alpha \in \hat{G}, f \in A .
$$

By repeated application of (3) and the definition of $T_{\alpha}$, we obtain $\mathfrak{F}\left(\phi T_{\alpha}(f)\right)(\tau(u))=\mathfrak{F}\left(T_{\alpha}(f)\right)(u)=\mathfrak{F}(f)(\alpha u)=\mathfrak{F}(\phi(f))(\tau(\alpha u))$. If we now apply (1), the last term above becomes $\mathfrak{F}(\phi(f))\left(\tau(\alpha) \tau(u)(\tau(e))^{-1}\right)$

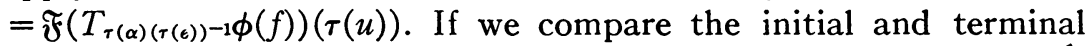
points of the string of equalities, and note that the range of $\tau$ is $\hat{G}$, we see that

$$
\mathfrak{F}\left(\phi T_{\alpha}(f)\right)=\mathfrak{F}\left(T_{\left.\tau(\alpha)(\tau(e))^{-1} \phi(f)\right)} .\right.
$$


Thus $\phi T_{\alpha}(f)=T_{\tau(\alpha)(\tau(e))-1} \phi(f)$. However $f$ is arbitrary in $A$, so $\phi T_{\alpha}$ $=T_{\tau(\alpha)(\tau(e))^{-1}}$ and thus we may take $\rho(\alpha)=\tau(\alpha)(\tau(e))^{-1}$. Hence $\phi$ satisfies (2), and (b) implies (c).

If the mapping $\phi$ is an involution on $A$, a result of Civin and Yood [1] yields a homeomorphism $\sigma$ of period two of $\hat{G}$ onto itself such that $\mathfrak{F}(\phi(f))(\sigma \alpha)=[\mathfrak{F}(f)(\alpha)]^{-}$. Methods totally similar to the above and to those of $[2 ; 4]$ allow one to establish the following theorem.

THEOREM 3. Let $A$ be the group algebra of a locally compact abelian group, and let $\phi$ be an involution on $A$. The following conditions are equivalent.

(a) $\phi$ is an isometry.

(b) The associated homeomorphism $\sigma$ of period two of $\hat{G}$ onto itself satisfies $\sigma(e) \sigma(x y)=\sigma(x) \sigma(y)$, for all $x, y \in \hat{G}$.

(c) $\phi$ satisfies (2).

\section{REFERENCES}

1. P. Civin and B. Yood, Involutions on Banach algebras, Pacific J. Math. vol. 9 (1959) pp. 415-436.

2. H. Helson, Isomorphisms of abelian group algebras, Ark. Mat. vol. 2 (1953) pp. 475-487.

3. C. E. Rickart, The uniqueness of norm problem for Banach algebras, Ann. of Math. vol. 51 (1950) pp. 615-628.

4. J. G. Wendel, On isometric isomorphism of group algebras, Pacific J. Math. vol. 1 (1951) pp. 305-311.

UNIVERSITY OF OREGON 